\title{
Cooperative Signaling with Soft Information Combining
}

\author{
Rui Lin, Philippa A. Martin, and Desmond P. Taylor \\ Department of Electrical and Computer Engineering, University of Canterbury, Christchurch 8014, New Zealand
}

Correspondence should be addressed to Rui Lin, rli24@student.canterbury.ac.nz

Received 30 August 2009; Accepted 27 October 2009

Academic Editor: Luca De Nardis

Copyright ( 2010 Rui Lin et al. This is an open access article distributed under the Creative Commons Attribution License, which permits unrestricted use, distribution, and reproduction in any medium, provided the original work is properly cited.

We propose a Decode-and-Forward (DF) scheme using distributed Turbo code (DTC) for a three-node (source, relay, and destination) wireless cooperative communication system. The relay decodes, then interleaves, and reencodes the decoded data. It then forwards the reencoded packet and its instantaneous receive SNR to the destination. The performances using both ideal and quantized SNR are studied. The destination uses a modified metric within a Turbo decoding algorithm to scale the soft information calculated for the relay code. The proposed scheme is simple to implement and performs well.

\section{Introduction}

Cooperative communication has been shown to provide diversity gains in systems with limited numbers of transmit antennas through the use of relay nodes. Here, we consider a three-node (triangle) wireless network consisting of a source $(S)$, relay $(R)$, and destination $(D)$ node. All nodes are assumed to be half duplex and equipped with one antenna. This network can potentially achieve maximum diversity order of two.

In this paper, we propose a simple DF scheme using a DTC, but no CRC code. The relay simply decodes, interleaves, re-encodes, and forwards all packets without checking whether or not they are correct. The relay also forwards its average receive SNR for the packet to the destination. In other words, the relay is always "on" with hard decision forwarding. Inspired by the cooperative maximum ratio combiner proposed in [1], we propose a modified decoding metric at the destination which scales the soft information calculated for the relayed code so that not only diversity order two but also coding gain can be achieved compared to both the selective and adaptive DF schemes proposed in [2].

The other forms of always "on" DF relaying protocols either forward analogue type signals or do hard switching between two protocols. For example, [3] forwards the decoded Log Likelihood Ratio (LLR) for each information bit at low SNR and [4] forwards the estimated reliability of each parity bit, both of which are analogue signals. The relay in [5] switches between DF and AF based on the result of the CRC test and the relay in [3] switches to coded cooperation in the high SNR region. The only other always "on" hard decision DF scheme, to the best of our knowledge, is [6], in which the average BER is forwarded to the destination. We note that the forwarded BER in [6] is not the BER of the systematic bits but is the BER of the entire coded bit stream. This BER is difficult to obtain in practice, particularly when a recursive systematic convolutional (RSC) code is used at the relay. The relay in [6] needs to use a MAP decoder not only to decode the systematic bit but also to estimate the BER for the entire coded packet. This significantly increases complexity at the relay. In contrast, the scheme proposed in this paper can use a Viterbi decoder at the relay which is simpler. The overhead caused by forwarding the receive SNR is only a few bits per packet (3 bits for QPSK), which is negligible considering the length of a packet.

\section{System Description}

The distances between nodes $(S-D, S-R$, and $R-D)$ are normalized against the source to destination $(S-D)$ distance, $d_{S D}=1$. The other two distances are always smaller than or equal to 1 with $d_{S R}+d_{R D}=1$. Transmission is organized in a packet by packet fashion. The transmission of each packet is divided into two stages, namely, broadcast and relay. The channels connecting all three nodes are modeled 
as quasistatic Rayleigh block fading channels, which are constant over the combined broadcast and relay stages for each packet and change independently between adjacent packet transmissions. The channel coefficient is modelled as $\rho=\sqrt{g} h$, where $h$ is a circularly symmetric complex random variable with zero mean and unit average power and $g$ is the channel gain which is related to the distance according to $g=1 / d^{v}$, where $v$ is the path-loss exponent.

During the broadcast stage, the source encodes a block of information bits using an RSC code and broadcasts it to the destination and relay. The destination delays decoding until the end of the relay stage. The relay decodes the broadcast message, then it interleaves the decoded bits, and re-encodes them using the same or a different RSC code. It forwards the encoded packet to the destination during the relaying stage (while the source stays silent). The corresponding received signals at the relay and destination for a single packet are given by

$$
\begin{gathered}
y_{S R}=\sqrt{g_{S R}} h_{S R} x_{S}+n_{S R}, \\
y_{S D}=\sqrt{g_{S D}} h_{S D} x_{S}+n_{S D}, \\
y_{R D}=\sqrt{g_{R D}} h_{R D} x_{R}+n_{R D},
\end{gathered}
$$

where $y_{p q}$ is the received signal vector at node $q$ sent by node $p, x_{p}$ is the encoded symbol vector at node $p$, and $n_{p q}$ is the additive white Gaussian noise (AWGN) vector with a variance of $N_{0} / 2$ per dimension at node $q$. Note that $x_{R}$ may contain errors due to the relay incorrectly estimating the information bits.

Along with the re-encoded packet, the relay forwards its receive SNR, $\mathrm{SNR}_{S R}=\left(g_{S R}\left|h_{S R}\right|^{2} E_{S}\right) / N_{0}$, to the destination, where $E_{s}$ is the average symbol energy transmitted from the source. In contrast, [1] assumes that the destination obtains $\mathrm{SNR}_{S R}$ via training. In addition, we investigate the scenario that the relay sends this information to the destination in a quantized form using a small number of bits. No CRC codes are used, which more than offset the throughput loss due to transmitting the quantized SNR. After receiving the message from the relay, the destination uses a Turbo algorithm to decode the coded packets it received during the broadcast and relay stages.

\section{Proposed Decoder Scaling}

We use the BCJR decoding algorithm to decode each component code at the destination. Note that the packet sent from the relay can contain errors from decoding the source transmission. As a result, we modify the transition probability used to decode $y_{R D}$ (but not $y_{S D}$ ).

The transition probability of a BCJR decoder [7] is

$$
\gamma_{j}\left(s^{\prime}, s\right)=P\left(u_{j}\right) \exp \left(\frac{-E_{s}}{N_{0}}\|Y-\rho \cdot X\|^{2}\right),
$$

where $u_{j}$ is the $j$ th information bit and $P\left(u_{j}\right)$ is its a priori probability. $X$ is the encoder output vector corresponding to the $j$ th input bit which causes the state change from $s^{\prime}$ to $s, Y$ is the corresponding received signal vector, and $E_{s} / N_{0}$ is the average symbol SNR. In order to take the error probability at the relay into account, we extend the idea of "cooperative maximum ratio combining" as proposed in [1] to redefine the calculation of $\gamma$ as

$$
\gamma_{j}\left(s^{\prime}, s\right)=P\left(u_{j}\right) \exp \left(\frac{-E_{s}}{N_{0}} \cdot \zeta \cdot\|Y-\rho \cdot X\|^{2}\right)
$$

where $\zeta=\min \left(\mathrm{SNR}_{S R}, \mathrm{SNR}_{R D}\right) / \mathrm{SNR}_{R D}$. When the $S-R$ link experiences a deep fade or is much weaker than the $R-D$ link, $\zeta \approx 0$. Then, the extrinsic information generated from this decoding process is nearly zero for each information bit. In this case, the packet sent from the relay has no or very limited impact both on decoding the packet sent from the source and in making the final hard decision. Hence, error propagation is mitigated. Note that the extension to more than one relay is trivial. The component decoder for the code received directly from node $S$ has $\zeta$ set to 1 .

It is worth emphasizing the difference between the proposed scheme and the cooperative maximum ratio combining proposed in [1]. Although they look similar to each other mathematically, the underlying principles are very different. The principle of maximum ratio combining (MRC) can only be applied to repetition-based protocols including Demodulation-and-Forward [1, 8, 9], Amplifyand-Forward protocols [2], and DF using a repetition code [6]. This is because this type of combining requires the direct and the relayed transmissions to send the same symbols (bits); so they can be combined at the destination to form the input for the channel decoder. As a result, if a Turbo code is used, it must be transmitted by the user. Since a Turbo code generally has lower rate than a component convolutional code, these schemes will have lower rate than our scheme.

On the other hand, in our scheme, due to the interleaver which results in incremental relaying, the MRC cannot be applied before the channel decoder at the destination as the parity bits (symbols) from the direct and relayed copies are different. Therefore, it can be viewed that the cooperative combining is performed on the soft information through the iterative process. Then, in principle, the relay can use arbitrary different modulations and codes from those used during the broadcast stage and, at the destination, the soft information generated from these two codes is properly weighted and combined to make the decisions for the information bits.

\section{Simulation Results and Discussion}

Our simulations use packets formed by encoding blocks of 500 information bits and $v=3$. The Turbo decoder uses 15 iterations. BCJR component decoders are used. An 8-state rate $1 / 2$ RSC code with generator polynomial $[1,17 / 15]_{8}$ is used at both the source and relay. QPSK modulation is considered.

For comparison purposes, we also considered the selective DF, adaptive DF [2], ideal DF, conventional DF and direct transmission schemes. The difference between 


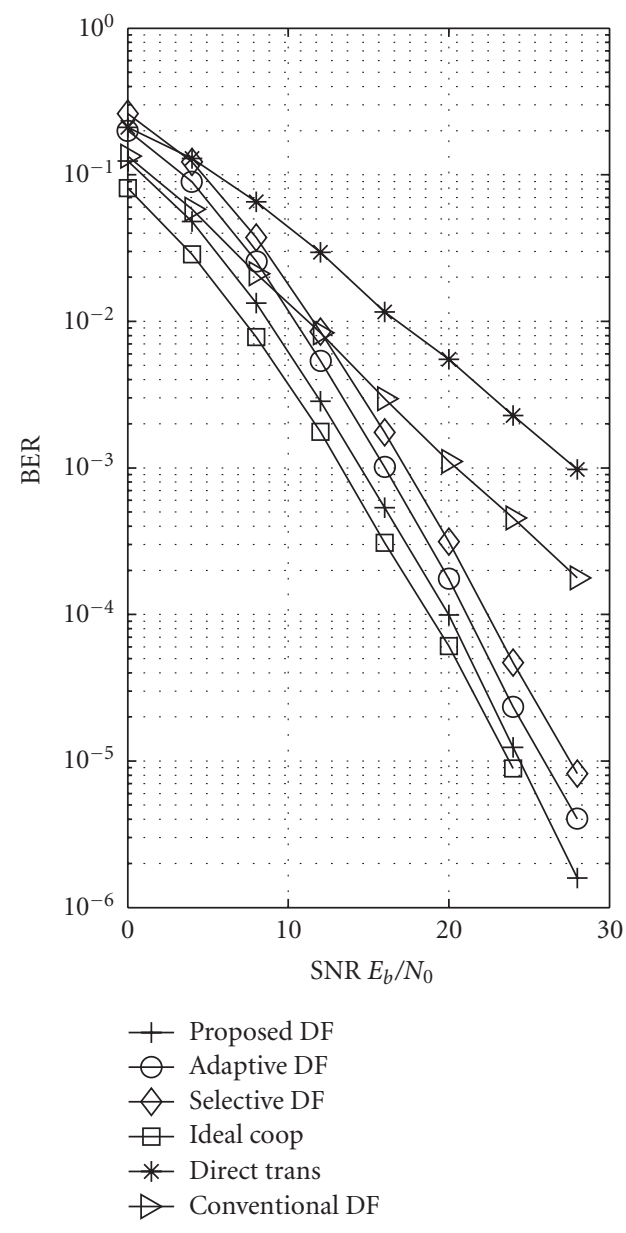

(a)

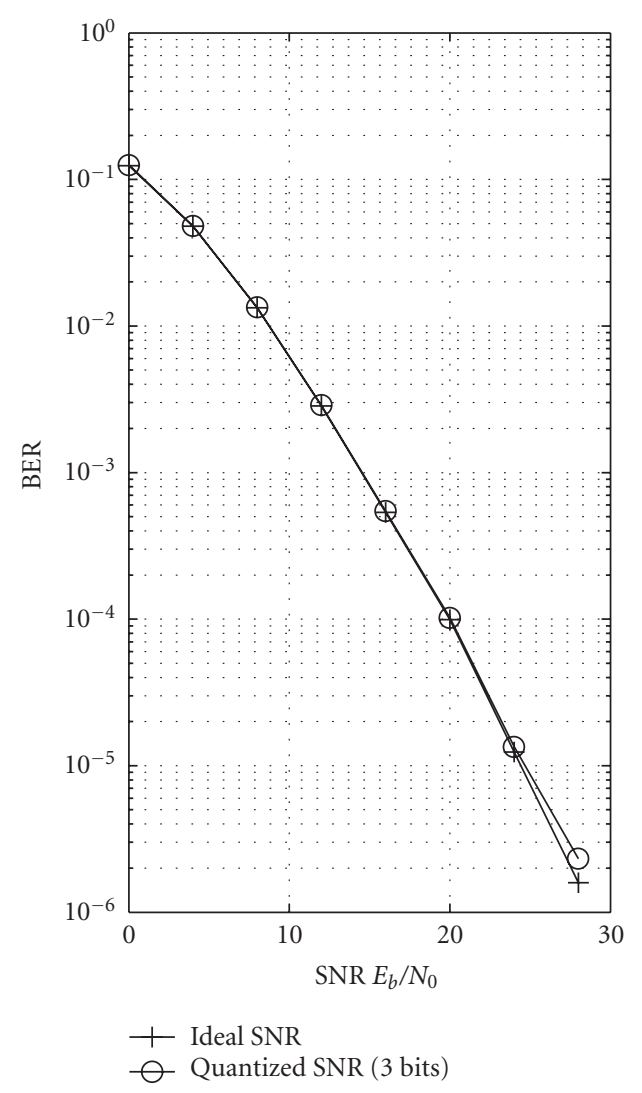

(b)

Figure 1: Performance curves $\left(d_{S R}=0.5, d_{R D}=0.5\right)$.

adaptive DF, and selective DF is that, when the CRC check is not satisfied, the source node transmits the second part of the code in adaptive DF during the relay stage, while there is no transmission during the relay stage in selective DF. Therefore, an $R-S$ feedback channel is required for adaptive DF. Ideal DF uses an error free $S-R$ channel while conventional DF uses the $S-R$ channel defined in (1). The destination treats the relayed packet as if it is error free in both ideal and conventional DF. Direct transmission is noncooperative and all the coded symbols are transmitted from the source node during both broadcast and relay stages. All the comparative schemes use the same Turbo code as the proposed scheme.

Figure 1(a) shows the performance of the above schemes when the relay is located at the mid-point between the source and destination. We can see that all the cooperative schemes, except conventional DF, achieve second-order diversity while direct transmission only achieves diversity order one. The proposed scheme achieves about $3 \mathrm{~dB}$ gain compared to selective DF and is only $1 \mathrm{~dB}$ worse than ideal DF.

Figure 1(b) shows that using 3 bits to quantize $S_{N R}$ can achieve almost identical performance to using ideal $\mathrm{SNR}_{S R}$. The SNR range being quantized is $-5 \mathrm{~dB}$ to $12.5 \mathrm{~dB}$. Each binary representation covers a $2.5 \mathrm{~dB}$ subrange. The mean of each subrange is used at the destination to calculate $\zeta$.

The position of the relay is moved to give $\left(d_{S R}=\right.$ $\left.0.7, d_{R D}=0.3\right)$ and $\left(d_{S R}=0.3, d_{R D}=0.7\right)$ in Figure 2 . We can see that the proposed scheme performs approximately the same as adaptive DF when the relay is close to the source but achieves performance gain as the relay moves towards the destination.

Based on the above observations, we may draw the following conclusion. When the $S-R-D$ channel is stronger than the $S-D$ channel, by properly scaling the soft information, we can improve performance using the $S-R-D$ channel even though the relay node may decode in error. We also observe that, for the proposed scheme, a relay located at the mid point between source and destination nodes gives the best performance. The proposed scheme performs well over a large range of $d_{S R}$.

For large networks, where a three-node triangle relay network is a building block, having simpler operations at the relay is highly desirable. In this sense, forwarding reliability information is obviously not very practical although it may perform better. This raises the question of how much performance is lost compared to soft information 


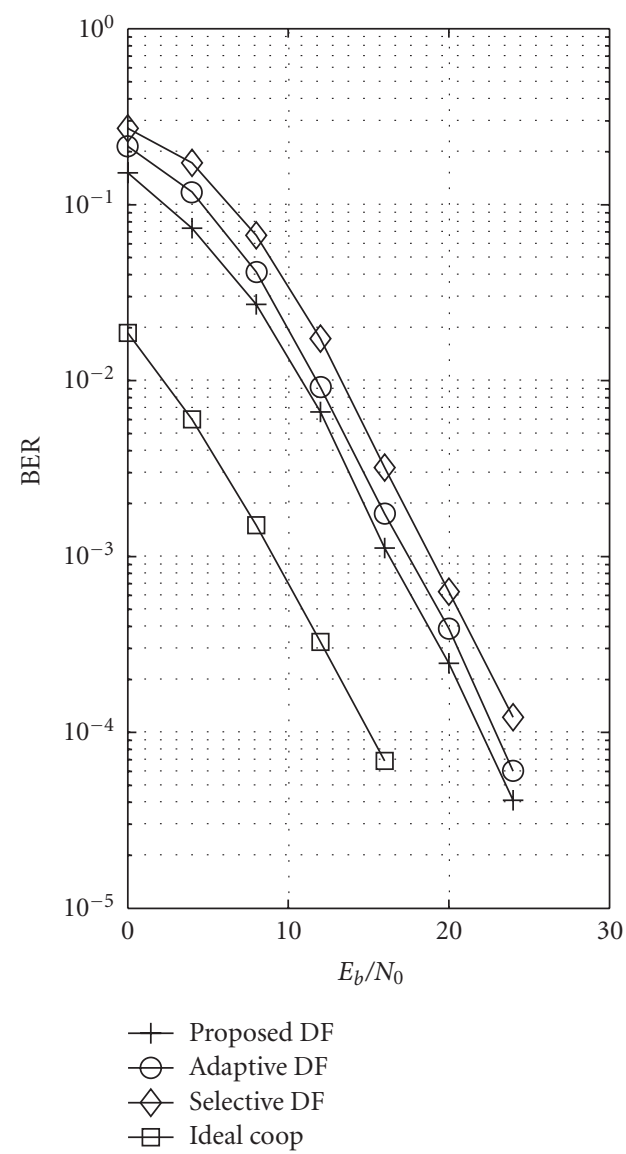

(a)

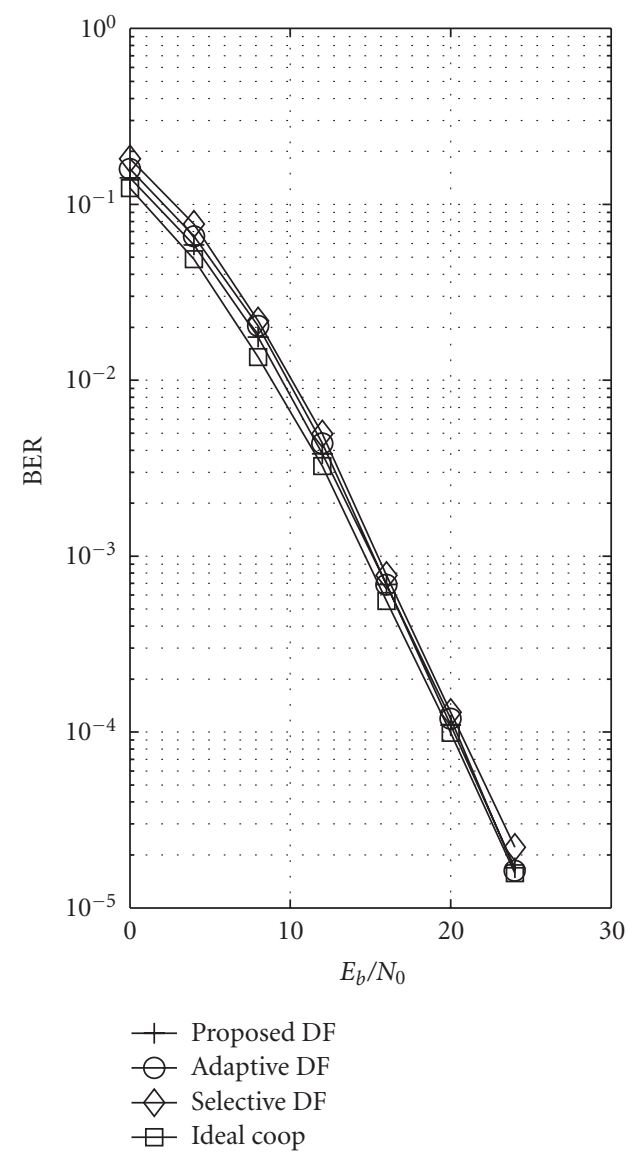

(b)

Figure 2: Performance curves when $\left(d_{S R}=0.7, d_{R D}=0.3\right)$ and $\left(d_{S R}=0.3, d_{R D}=0.7\right)$.

forwarding. Finding this loss analytically is beyond the scope of this paper and is a part of this ongoing research. However, based on the simulation results, we conjecture that this performance loss is very small if the relay is carefully chosen (e.g., $d_{S R} \leq 0.5$ ) because the performance gap between the proposed scheme and ideal cooperation is small.

\section{Conclusions and Further Work}

In this paper, we have proposed a simple DF scheme that not only achieves full diversity gain but also offers significant coding gain compared to the DF schemes of [2] for a wide range of $d_{S R}$ without significantly increasing complexity. The simulation results also show that quantizing the $\mathrm{SNR}_{S R}$ using 3 bits can achieve almost identical performance to using ideal $\mathrm{SNR}_{S R}$. This proposed scheme offers a practical solution to a triangle relay network and gives the designer significantly more freedom in system design (number of relays or using different codes). We intend as part of our ongoing research to carry out more detailed analysis of this scheme and to extend it to larger networks. This analytical work may lead to the finding of the optimal combining coefficient for the distributed Turbo coding.

\section{References}

[1] T. Wang, A. Cano, G. B. Giannakis, and J. N. Laneman, "High-performance cooperative demodulation with decodeand-forward relays," IEEE Transactions on Communications, vol. 55, no. 7, pp. 1427-1438, 2007.

[2] J. N. Laneman, Cooperative diversity in wireless networks: algorithm and architectures, Ph.D. thesis, MIT Press, Cambridge, Mass, USA, 2002.

[3] X. Bao and J. Li, "Efficient message relaying for wireless user cooperation: Decode-Amplify-Forward (DAF) and hybrid DAF and coded-cooperation," IEEE Transactions on Wireless Communications, vol. 6, no. 11, pp. 3975-3984, 2007.

[4] Y. Li, B. Vucetic, T. F. Wong, and M. Dohler, "Distributed turbo coding with soft information relaying in multihop relay networks," IEEE Journal on Selected Areas in Communications, vol. 24, no. 11, pp. 2040-2050, 2006.

[5] T. Q. Duong and H.-J. Zepernick, "On the performance gain of hybrid decode-amplify-forward cooperative communications," EURASIP Journal on Wireless Communications and Networking, vol. 2009, Article ID 479463, 10 pages, 2009.

[6] K. Lee and L. Hanzo, "Iterative detection and decoding for hard-decision forwarding aided cooperative spatial multiplexing," in Proceedings of IEEE International Conference on Communications (ICC '09), Dresden, Germany, June 2009.

[7] S. Lin and J. D. J. Costello, Error Control Coding, Prentice-Hall, Upper Saddle River, NJ, USA, 2nd edition, 2004. 
[8] D. Chen and J. N. Laneman, "Modulation and demodulation for cooperative diversity in wireless systems," IEEE Transactions on Wireless Communications, vol. 5, no. 7, pp. 1785-1794, 2006.

[9] M. Benjillali and L. Szczecinski, "A simple detect-and-forward scheme in fading channels," IEEE Communications Letters, vol. 13, no. 5, pp. 309-311, 2009. 

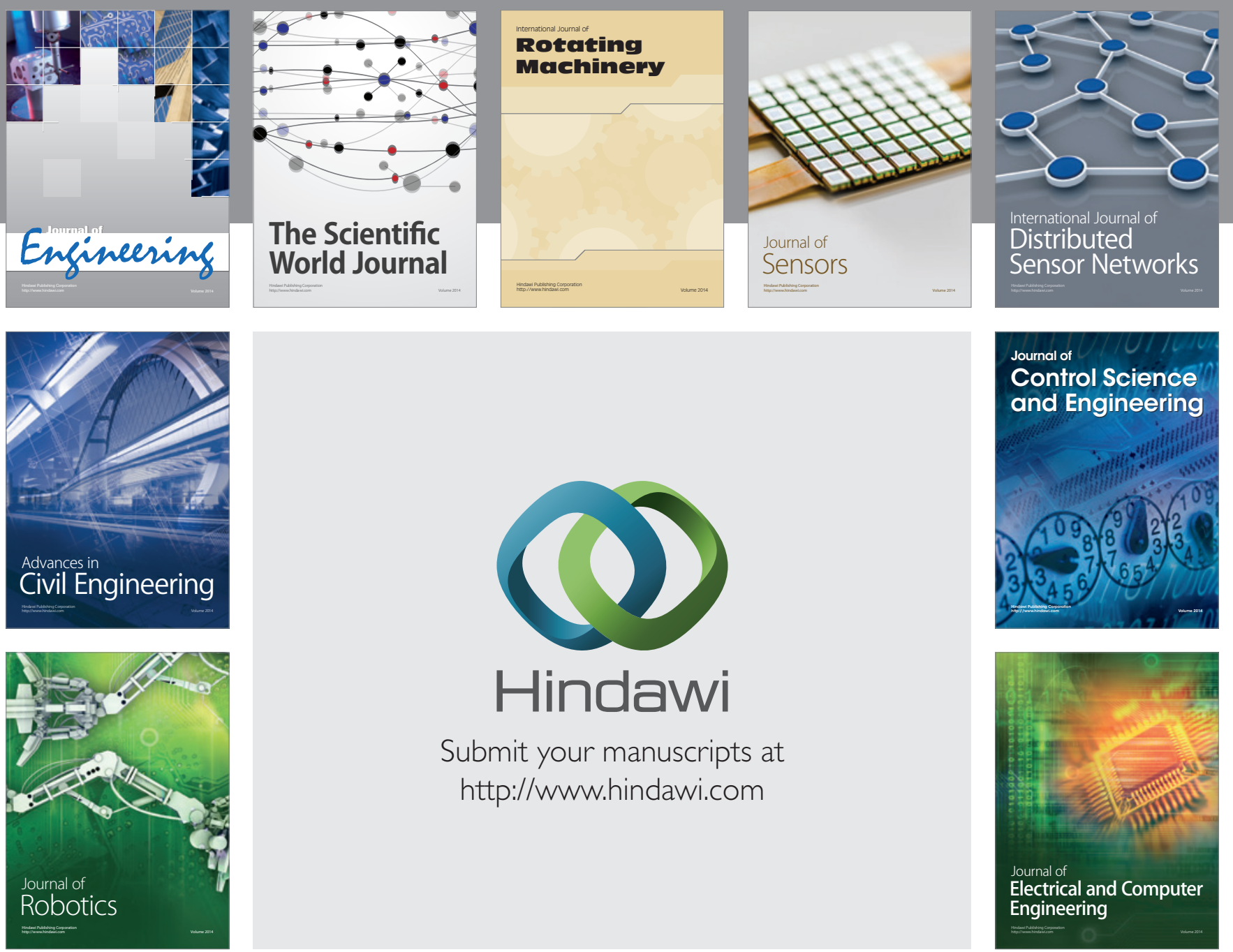

Submit your manuscripts at

http://www.hindawi.com
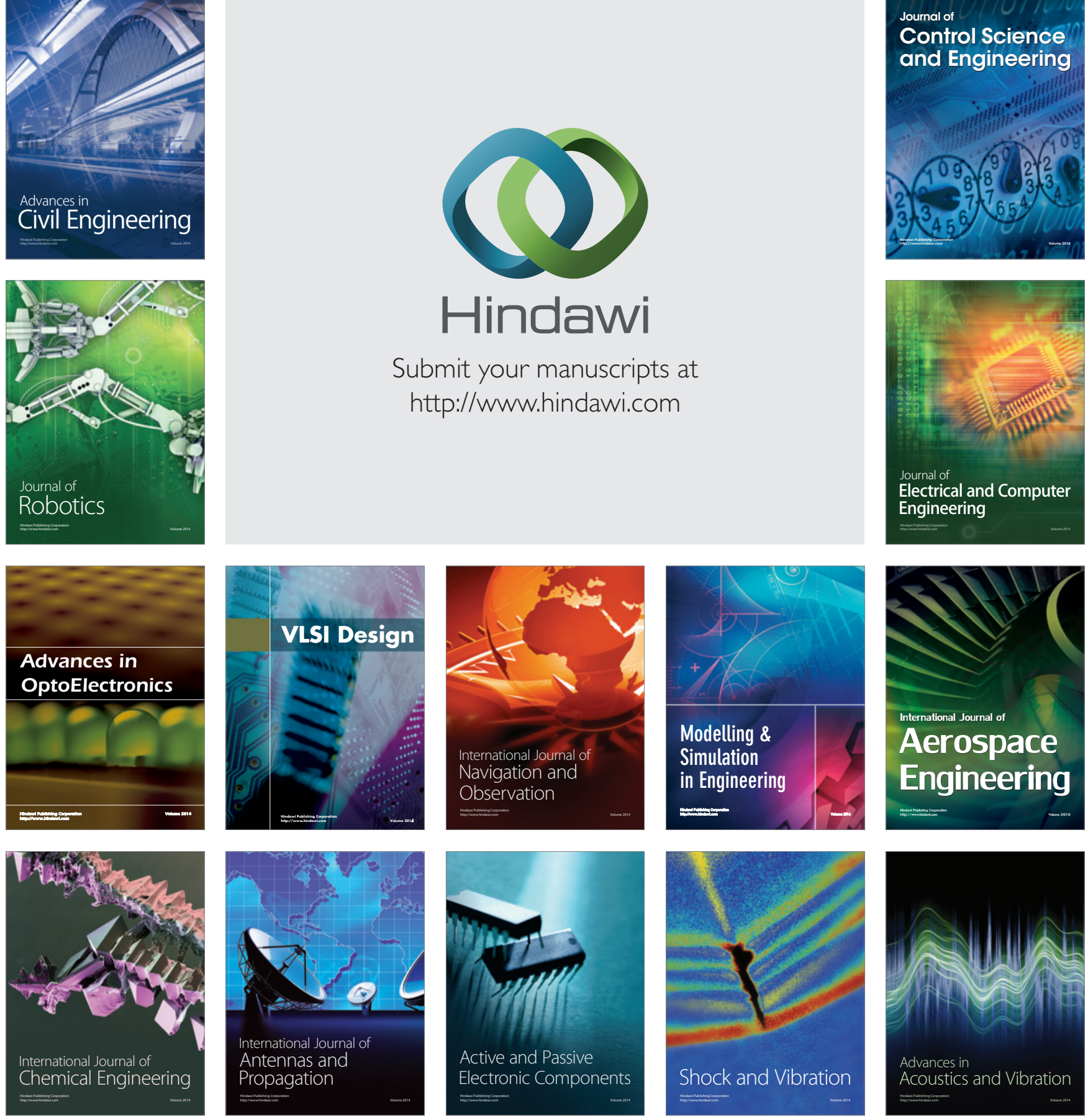